\title{
A Genetic Algorithm for Face Fitting
}

\author{
David Hunter ${ }^{1}$, Bernard P. Tiddeman ${ }^{2}$ and David I. Perrett ${ }^{1}$ \\ ${ }^{1}$ School of Psychology, University of St Andrews, Fife, Scotland, UK \\ ${ }^{2}$ Department of Computing, University of Aberystwyth, Ceredigion, Wales, UK \\ $\{d w h 5, d p\} @ s t-a n d r e w s . a c . u k, b p t @ a b e r . a c . u k$
}

Keywords: Genetic Algorithms, Face Fitting, Three Dimensional Morphable Models

\begin{abstract}
Accurate estimation of the shape of human faces has many applications from the movie industry to psychological research. One well known method is to fit a Three Dimensional Morphable Model to a target image. This method is attractive as the faces it constructs are already projected onto an orthogonal basis making further manipulation and analysis easier. To date use of Morphable Models have been limited by the inaccuracy and inconvenience of current face-fitting methods. We present a method based on Genetic Algorithms that avoid the local minima and gradient image errors that current methods suffer from. It has the added advantage of requiring no manual interaction to initialise or guide the fitting process.
\end{abstract}

\section{INTRODUCTION}

Accurate analysis of the 3D shape of human faces has been limited by the relative lack of data from three-dimensional scanners. Databases of 2D face images are often far more complete, easier to produce, and have a long history that pre-dates 3D scanners. However accurate shape estimating using landmarks, or other measures, is limited by problems of pose and orientation. Blanz and Vetter proposed a solution in the form of a Morphable Model that captures in a statistical model the space of human face shapes and then attempts to find from this face-space a model that most closely matches the target image (Blanz and Vetter, 1999). The advantage of this algorithm is that shape can be estimated from a far wider variety of poses than with a two-dimensional method such as the commonly used AAM (Cootes et al., 1998). Also, illumination is less of an issue as the illumination of the three-dimensional model can be computed by physical simulation. Widespread adoption of these models has been hampered by the lack of accuracy in the fitting of the model.

Most current methods involve minimizing a cost function based on the $L^{2}$-norm between a rendered face model with a particular set of parameters and a target image. As the derivatives of this function can be approximated, many previous authors have used gradient descent methods. However these methods are prone to local-minima problems. Also the derivatives are approximated and are only valid if the face is al- ready closely aligned with the target image. These derivatives introduce a new source of error to the fitting that is most pronounced when the gradient is shallow, as well as a windowing effect that makes it difficult to detect shape updates that differ significantly in scale.

We used an alternative minimization approach that avoids many of the problems associated with gradient descent, called a Genetic Algorithm. This algorithm uses the 'best' results from the previous iteration to seed a new set of trial parameters. This allows a greater proportion of the parameters' space to be analysed and so is less likely to become 'stuck' in a local-minima and so find a global optimum.

\section{Background}

In their original paper on Morphable Models, Blanz and Vetter used a stochastic gradient descent method to minimize the $L^{2}$-norm between a synthesized face image and a target image (Blanz and Vetter, 1999). A significant contribution to the field of 3D face fitting was made by Romdhani who together with Blanz and Vetter investigated estimation of shape changes via optical-flow (Romdhani et al., 2002). They also adapted a version of the inverse Lucas Kanade algorithm (Baker and Matthews, 2004) for 3D face models (Romdhani and Vetter, 2003). They found this method efficient at estimating face shapes provided no change in pose was detected, in which 
case the derivative images would have to recalculated in their entirety. The most promising was a multifeature fitting strategy that combined, in a Bayesian fashion, a set of different differentiable cost functions designed to extract different aspects of the image; for example, edges and particular illumination artefacts such as specular reflection (Romdhani, 2005). Like previous methods, these functions were differentiable and required a good initial estimate of parameters. Faggian et al. adapted the method for multiple views of the same face, however we will be working with just one view (Faggian et al., 2008).

Xiao et al. used a 2D to 3D method whereby an Active Appearance Model was constructed from a 3DMM. Thus methods developed to fit and track AAMs can be used with 3D models. However the combined model also spans a large set of parameter values that result in invalid 3D shape models (Xiao et al., 2004). These methods all suffer from both the local-minima and windowing problems described above.

Fitting a model by matching it to prominent features in the target image is an appealing option. The most obvious of these are the boundaries such as those between the face and background and internal boundaries such as the edges of eyes, the mouth etc. Moghaddam et al. used face silhouette taken of the same source from multiple angles to capture a 3DMM. They used an XOR based cost function where a high cost was applied to silhouette edge points that are found in one image but not at the equivalent point in the other. Not all the boundaries on the images and models are appropriate for fitting. Hair, for example, provides false edges, and the model itself can provide false silhouettes as it is defined over the face only and not the full head. The cost function was therefore weighted towards appropriate silhouettes (Moghaddam et al., 2003).

A number of techniques make use of shape-fromshading, solving a partial differential equation linking the image intensity to the reflectance map based on the assumption that the surface is Lambertian. Patel and Smith estimated the 3D shape by minimising the arc-distance between the surface normal of the Morphable Model and the illumination cone. These constraints applied only to vertex points and as such allowed the shape-from-shading model to capture finescale surface details. Current Shape from shading formulations rely on specific lighting and camera setups, for example a distant light source or a light source at the optical centre of the image. This constraint is not present when the lighting model is calculated by physical simulation (Patel and Smith, 2009).

\section{Constructing a Morphable Model}

Three dimensional Morphable Models, introduced by Blanz and Vetter, use Principle Components Analysis to describe the space of human faces as a set of orthogonal basis vectors. Given a set of 3D dimensional face models we find a set of one-to-one correspondences between vertices by delineating key points on the models, such as eyes, nose, mouth etc. The exemplar is warped into alignment with the target face using the landmarks to drive a 3D thin-plane-spline model. Correspondences between face models and an exemplar face model are found by casting rays out from the vertices of the exemplar models in the direction of the surface normal at the vertex, the position on the target model intersected by the ray is considered to be the corresponding vertex. The meshes are remapped by warping the vertices of the exemplar mesh to the corresponding vertices of the target mesh, thus creating a new mesh with the vertex count and structure of the exemplar and the shape of the target mesh. Colour is warped similarly using the correspondences defined to between the two shapes. We concatenate the resulting vertex positions and colour values as,

$$
\begin{gathered}
\mathbf{s}=\left(x_{1}, y_{1}, z_{1}, x_{2}, y_{2}, z_{2}, \cdots, x_{n}, y_{n}, z_{n}\right)^{T}, \\
\mathbf{t}=\left(r_{1}, g_{1}, b_{1}, r_{2}, b_{2}, g_{2}, \cdots, r_{m}, g_{m}, b_{m}\right)^{T}
\end{gathered}
$$

Each face is centred by subtracting the mean of all the faces and PCA performed. A reduced set of 40 eigenvectors for each of shape and colour were used to describe the face space. The shape $s$ and colour $t$ of a new face are generated as a linear combination of weighted PCA vectors $\mathbf{s}_{j}, \mathbf{t}_{j}$ and the averages $\hat{\mathbf{s}}$ and $\hat{\mathbf{t}}$.

$$
\mathbf{s}=\hat{\mathbf{s}}+\sum_{j=1}^{k} \alpha_{j} \mathbf{s}_{j}, \mathbf{t}=\hat{\mathbf{t}}+\sum_{j=1}^{k} \beta_{j} \mathbf{t}_{j}
$$

With the probability distribution over the PCA facespace defined as

$$
p(\mathbf{s}) \approx e^{-\frac{1}{2} \sum_{i} \frac{\alpha_{i}^{2}}{\sigma_{\mathbf{s}, i}}}
$$

where $\sigma_{\mathbf{s}, i}$ is the standard deviation of the $i^{t h}$ shape component. The PDF for colour is defined similarly.

The weights $\alpha_{j}$ and $\beta_{j}$ form the parameter vectors $\alpha$ and $\beta$. New faces are created by varying these parameters. In order to render the model a set of camera parameters specifying the position, pose and scale of the face relative to a camera position are required. In the rest of this paper we will be referring to the concatenated shape and colour parameters $\alpha, \beta$, together with the camera parameters, as the Morphable Model with parameters $\mathbf{p}$. The image of the rendered Morphable Model with parameters $\mathbf{p}$ is denoted $\mathcal{M}(\mathbf{p})$. This process is described in more detail in (Blanz and Vetter, 1999). 


\section{Genetic Algorithm}

\subsection{Cost function}

In order to extract three dimensional facial features we minimize the $L^{2}$-norm of the difference between a rendered $3 \mathrm{D}$ face model and the target image.

$$
C(\mathbf{p})_{l}=\frac{1}{|\Omega|} \sum_{\mathbf{x} \in \Omega}\left(\mathcal{M}(\mathbf{p})_{l a b}-I_{l a b}\right)^{2}
$$

where $\Omega$ is the subset of all samples in the image covered by the rendered face image and $|\Omega|$ is the number of samples in $\Omega$. The cost function is scaled to the number of rendered samples to avoid degenerate minimisation.

The calculation is performed in $\mathrm{L}^{*} \mathrm{a} * \mathrm{~b} *$ space as this allows an emphasis on the Intensity of the image (the $\mathrm{L}^{*}$ values are on average larger than the $\mathrm{a}^{*}$ and $b^{*}$ ) with some colour information included in the model.

We found that fitting to an $L^{2}$-norm alone was unsatisfactory as the model tended to have difficulty matching the edges of the face. The cost function is unspecified outside the area of the rendered model so the edges of the face are generally undefined by this method. We added an edge fitting metric as defined by (Romdhani, 2005). A Sobel edge detector is used to find edges in the target image. For each position in the target image the distance to the nearest edge point is found. The error metric for the edge detector becomes

$$
C(\mathbf{p})_{e}=\frac{1}{\Psi_{\mathcal{M}}(\mathbf{p})} \sum_{x \in \Psi_{\mathcal{M}}(\mathbf{p})}\left(\underset{x}{\operatorname{x}} \underset{x}{\operatorname{argmin}}\left|x-\Psi_{I}\right|_{2}^{2}\right)
$$

$\Psi_{I}$ and $\Psi_{\mathcal{M}}(\mathbf{p})$ denotes the set of detected edge points after a Sobel edge detector has been applied to the target image and rendered Morphable Model respectively.

The error metrics are combined into a single cost function

$$
C(\mathbf{p})=\lambda C(\mathbf{p})_{l}+\mu C(\mathbf{p})_{e}
$$

Where $\lambda$ and $\mu$ were chosen to be 1 and 15 respectively. These numbers were chosen such that the ratio $\frac{\lambda}{\mu}=\frac{\text { median }\left(C_{l}\right)}{\text { median }\left(C_{e}\right)}$. The medians of $C_{l}$ and $C_{e}$ were estimated empirically by rendering a set of Morphable Models with random parameters and estimating the respective error metrics between them and a randomly selected target image.

\subsection{Minimisation using a Genetic Algorithm}

The minimization method employs a standard genetic algorithm. There are many books and papers on this topic, and just as many variations on the basic method. For completeness we will outline the exact method used, much of which is from the book 'Essentials of Meta-heuristics' (Luke, 2009). The algorithm is inspired by Darwinian evolution aims find the global minimum by 'breeding' an optimal individual. Each generation, i.e. iteration of the algorithm, a population of possible solutions is evaluated and using the cost function (equation (7)). The best solutions are kept and the parameters combined to create a new population for the next iteration of the algorithm. In this manner the algorithm converges to the global optimum, the parameters of which are the values of $\mathbf{p}$ that minimise equation (7).

The algorithm begins by generating a set of samples from a distribution believed to contain the global minima of an cost function. In the selection phase a cost function is applied to this set of samples and the $m$ best selected as 'parents.' A new set of samples are generated from the parent set by selecting random pairs of parents and combining their parameters, this is known as Cross-Over. In a final Mutation phase the combined pairs are randomly altered to introduce variation into the population. The cost function is applied to these child samples and the $m$ best become the 'parents' of the next generation. The algorithm repeats until no further improvement is made over a pre-defined number of generations.

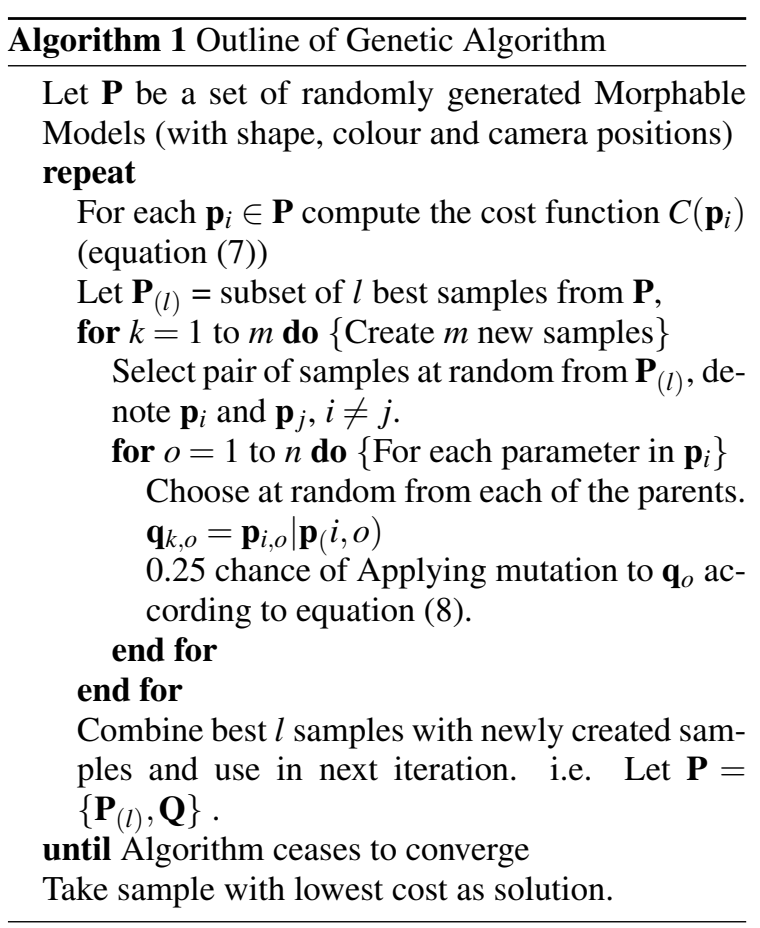

Selection Each subject in the current generation is evaluated using the fitness function $C$ and a subset 
of 25 with the 'best,'i.e. lowest, scores selected as parents for the next generation. These 'parents' survive into the next generation and are randomly paired to produce offspring.

Cross-Over In order to create a new 'child' subject from two parents it is necessary to select individual features from one parent or another, in the hope that the 'child' will feature the best parameters from each parent. As we do not know in advance which features offer the best improvement we select the features at random. Thus, for each parameter $i$ in the parameter vector $\mathbf{p}$ we select, randomly, one of the two parents and copy that parents parameter. In our implementation we did not bias towards either parent and thus each parameter has a 0.5 chance of being selected from each parent.

Mutation The offspring that result from the Selection and Cross-over will differ from their parents and uniqueness is enforced, however only parameter values randomly generated in the initialisation phase will be explored. In order that the parameter space is adequately explored by the algorithm, each parameter has a chance of being mutated, the probability of mutation is known as the mutation factor. A trivial implementation of the mutation would be to add a random amount to the parameter determined by the Probability Density Function (see equation (4)) for the parameter. However this adversely affects the convergence time of the algorithm as the parameters will frequently be far from the global optimum. To avoid this we used a new method that constrained the new mutated value to be with-in or close to the area that the population is converging towards. We assume that the two 'parent' values, being chosen from the amongst the best current solutions, frame the global minimum and thus we concentrate our search between these two values. This is a trade-off between a search that covers the whole space of possible Morphable Models and the speed of convergence. Define the mutated parameters $\mathbf{p}^{\prime}$ as

$$
\begin{gathered}
\mathbf{p}_{i}^{\prime}=\frac{1}{2}\left(p^{(1)}+p^{(2)}\right)-\rho+r \\
r \in \mathcal{U}\left(-\frac{1}{2}\left(p^{(1)}+p^{(2)}\right), \frac{1}{2}\left(p^{(1)}+p^{(2)}\right)\right) \rho
\end{gathered}
$$

where $p^{(1)}$ and $p^{(2)}$ are the parameters of the two selected parents and $\rho$ is a constant that allows the value of the new parameter $p^{\prime}$ to randomly stray beyond the limits described by $p^{(1)}$ and $p^{(2)}$, preventing the algorithm from becoming too constrained. In our system $\rho=1.2$. $\mathcal{U}(a, b)$ is the uniform distribution defined over the range $a$ to $b$ inclusive.

Elitism The best result from the previous generation is preserved in the new generation. This makes the search similar to a 'down-hill' search.

\section{Results}

3D models of 185 individuals (123 females, 62 males) of student age (17-23 years) were captured using a Cyberware scanner. A Morphable Model was constructed using these heads as outlined in section 3 . Further, 43 photographs of female subjects, also student aged were taken. These photographs were taken under controlled lighting conditions; these conditions were different from the lighting conditions of the $3 \mathrm{D}$ capturing system.

Shape estimation of each of the 43 subjects was carried out using both the Genetic-Algorithm method, outlined above, and using a Taylor-Series gradient descent method similar to (Blanz and Vetter, 1999). For the Taylor-Series method the faces where initialized by hand, placing a Morphable Model in the average configuration in the location on the face that most closely matched the subjects own face. To overcome the windowing problem we used a multi-scale fitting strategy.

Results of fitting using our GA algorithm are shown in figures 1 and 3 .

In order to get some empirical measures we would ideally like to have a three-dimensional face model that exactly matches the photographic image for comparison. As we have no access to such models we opted for a feature point matching strategy. Each of the 43 photographic images were hand delineated, marking out clear feature points, e.g. corner of eyes, mouth, chin etc. Identical landmarks were found on the Morphable Model, and appropriate shape updates found such that the landmarks were appropriately adjusted when the Morphable Models parameters were updates. When fitting, either by the Taylor-series method or the Genetic Algorithm, is completed the landmarks are updated to match the Morphable Model and projected onto the two-dimensional image. Each landmark is compared with its corresponding handplaced landmark to determine the accuracy of the fitting in an $L^{2}$, least squares sense.

$$
\chi^{2}=\sum_{i}\left|l_{i}-M\left[\hat{s}_{i}+\sum_{j=1}^{k} \alpha_{j} s_{i, j}\right]\right|_{2}^{2}
$$

Here $l_{i}$ is the 2D position of the $i^{\text {th }}$ hand-placed landmark. $\hat{s}_{i}$ denotes the position in the 3D shape average 


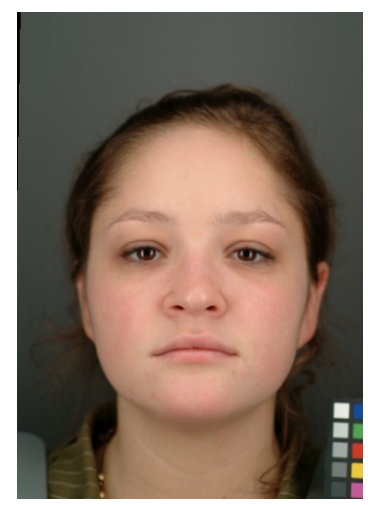

(a)

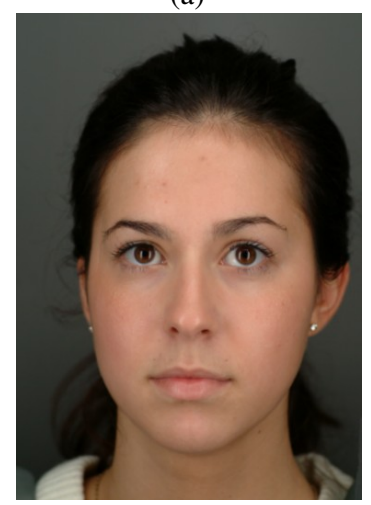

(c)

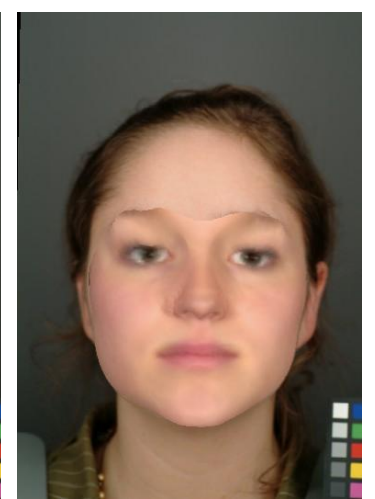

(b)

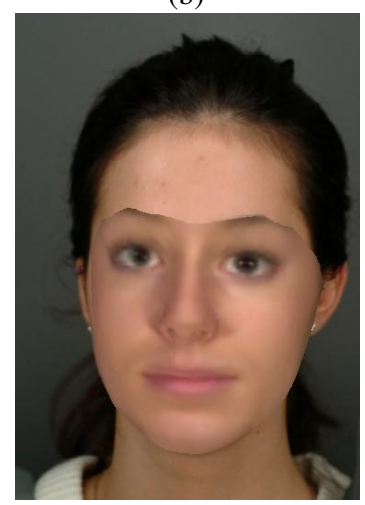

(d)
Figure 1: Example results of the GA face fitting algorithm. The left column shows the original subjects the right column the rendered shape estimation that approximately minimises the cost function $C$. The right most image shows the final result of the algorithm.

and $s_{j}, i$ the shape update of the $i^{t h}$ landmark in the $j^{t h}$ principal shape component. $M$ is a linear transform from $3 \mathrm{D}$ to $2 \mathrm{D}$ built using the camera parameters.

Table 1 shows the results of fitting to 43 example images using both the Taylor-Series and Genetic Algorithm methods. From these results we can see that the GA method offers a clear improvement over the Taylor-Series method, the difference is significant to $\mathrm{p}=0.005$, using a single-tailed paired t-test.

\begin{tabular}{|c|c|c|}
\hline Method & Mean & Standard Deviation \\
\hline Taylor-Series & 44.0 & 12.9 \\
GA & 38.8 & 4.5 \\
\hline
\end{tabular}

Table 1: Average error from template fitting computed as mean squared difference in pixels between landmark pairs. The images are 378 by 478 pixels. The results are averaged across 43 fitted images.

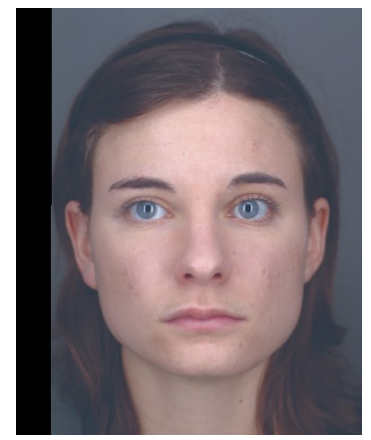

(a) Original Image

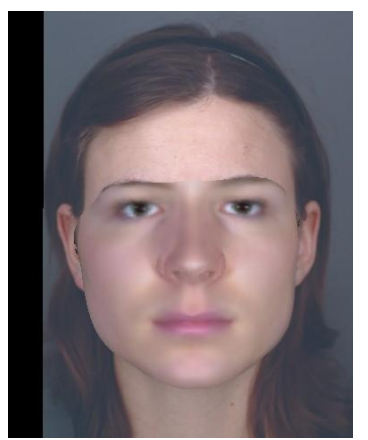

(b) GA Fitted Image

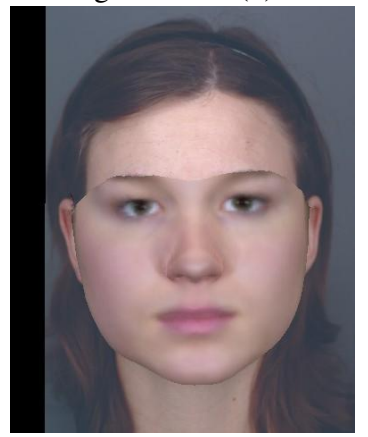

(c) Taylor-Series Fitted Image
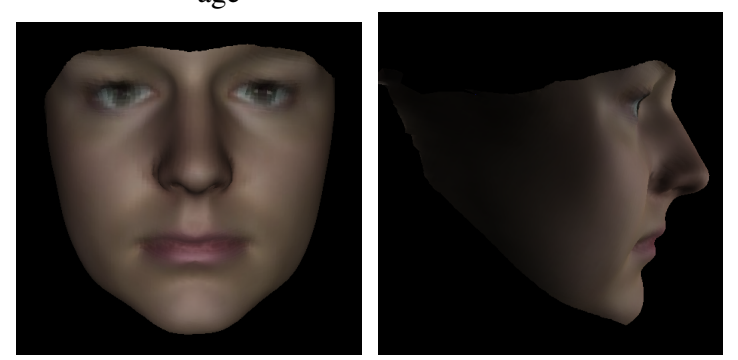

(d) Model only - Front View (e) Model only - Profile View

Figure 3: Example results from the face fitting algorithms. The top-left image shows the target face image for one of the subjects. The top right image is the result of the Genetic Algorithm applied to the target image. The middle row shows the results of fitting using the Taylor-Series algorithm for comparison. On the bottom row the models produced by the Genetic Algorithm are shown in both full-face (left) and profile (right) views.

\section{Conclusion}

Previous authors have either evaluated the results by visual inspection or by using the algorithm to identify an individual from a set of images (Romdhani et al., 2002; Patel and Smith, 2009). As far as we are aware we the first to have attempted to evaluate the accuracy of the fitting independently of the cost function, albeit limited to $2 \mathrm{D}$ projection rather than using a specific target model. 


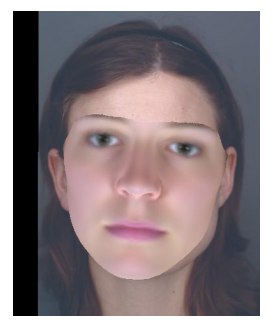

(a) $1^{\text {st }}$ iteration

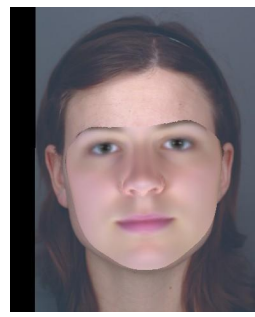

(b) $3^{\text {rd }}$ iteration

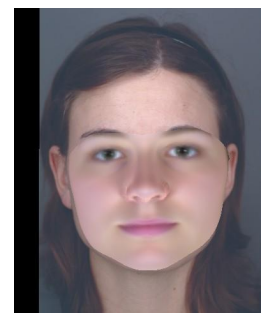

(c) $5^{\text {th }}$ iteration

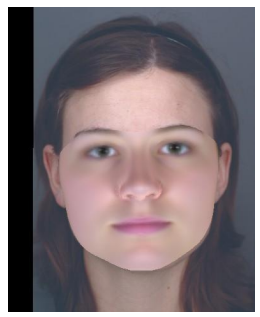

(d) $11^{\text {th }}$ iteration

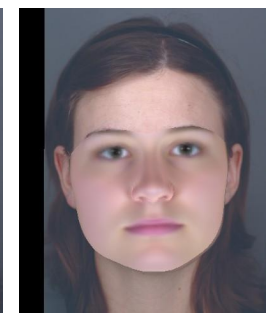

(e) $28^{\text {th }}$ iteration

Figure 2: The progress of the Genetic Algorithm in fitting to an example face. Each image shows the best sample from the indicated iteration.

The algorithm described offers a clear improvement over the simple Taylor-Series method. The Genetic Algorithm is able to reasonably accurately estimate the shape of the face without guidance by feature landmarks or other form of initialization. We believe this offers a significant improvement over current techniques as the method can be applied easily to large data-sets. One drawback of the algorithm that is worth mentioning is the speed. The average time taken for each subject in our set was 18.4 minutes on a $2.4 \mathrm{GHz}$ Intel(R) Core(TM)2 CPU. Gradient descent methods are significantly faster, taking an average of 4.7 minutes each. Although slower our method is more accurate than the Taylor-Series method. The standard deviation of the errors is significantly larger for the Taylor-Series method as this method produces highly inaccurate fits in a number of cases, whereas the GA method is more consistent.

Rather than implement exactly some of the stateof-the-art techniques we have used a simplified version that distils the various algorithms down to their essence as iterative gradient descent methods. Some of the methods such as (Romdhani and Vetter, 2003) and (Xiao et al., 2004) which attempt to exchange accuracy for speed are not considered as accuracy is our main aim. At the other end of the spectrum the multi-features fitting strategy of Romdhani's thesis uses many different error metrics in combination to produce a face model (Romdhani, 2005). We have not attempted use all of these metrics in our comparison, however we believe that they are likely to exhibit many of the same problems as the Taylor-Series method. This is due to the problems of local-minima and errors in derivative calculation, a problem inherent in gradient descent techniques. It is also worth noting that both Romdhani's algorithm (Romdhani, 2005) and (Blanz and Vetter, 1999) both rely on manual placement of landmarks on each face image both initialise and to guide the fitting. In this respect our method provides a clear advantage in that no landmark placement is required.

\section{REFERENCES}

Baker, S. and Matthews, I. (2004). Lucas-kanade 20 years on: A unifying framework. International Journal of Computer Vision, 56(1):221 - 255.

Blanz, V. and Vetter, T. (1999). A morphable model for the synthesis of $3 \mathrm{~d}$ faces. In SIGGRAPH '99: Proceedings of the 26th annual conference on Computer graphics and interactive techniques, pages 187-194, New York, NY, USA. ACM Press/Addison-Wesley Publishing Co.

Cootes, T. F., Edwards, G. J., and Taylor, C. J. (1998). Active appearance models. Lecture Notes in Computer Science, 1407:484-.

Faggian, N., Paplinski, A. P., and Sherrah, J. (2008). 3d morphable model fitting from multiple views. In $F G$, pages 1-6. IEEE.

Luke, S. (2009). Essentials of Metaheuristics. Lulu. Available for free at http://cs.gmu.edu/ sean/book/metaheuristics/.

Moghaddam, B., Lee, J., Pfister, H., and Machiraju, R. (2003). Model-based 3d face capture with shapefrom-silhouettes. In In IEEE International Workshop on Analysis and Modeling of Faces and Gestures, page pages.

Patel, A. and Smith, W. A. P. (2009). Shape-from-shading driven $3 \mathrm{~d}$ morphable models for illumination insensitive face recognition. In $B M V C$. British Machine Vision Association.

Romdhani, S. (2005). Face Image Analysis using a Multiple Feature Fitting Strategy. $\mathrm{PhD}$ thesis, University of Basel.

Romdhani, S., Blanz, V., and Vetter, T. (2002). Face identification by fitting a $3 \mathrm{~d}$ morphable model using linear shape and texture error functions. In Computer Vision-ECCV'02, volume 4, pages 3-19, Copenhagen, Denmark.

Romdhani, S. and Vetter, T. (2003). Efficient, robust and accurate fitting of a $3 \mathrm{~d}$ morphable model. In 9th IEEE International Conference on Computer Vision (ICCV), pages 59-66.

Xiao, J., Baker, S., Matthews, I., and Kanade, T. (2004). Real-time combined $2 \mathrm{~d}+3 \mathrm{~d}$ active appearance models. In Proceedings of the IEEE Conference on Computer Vision and Pattern Recognition, volume 2, pages 535 -542 . 